[0212-7199 (2007) 24: 12; pp. 591-594] ANALES DE MEDICINA INTERNA Copyright $(\mathcal{C} 2007$ ARAN EDICIONES, S.L.

AN. MED. INTERNA (Madrid) Vol. 24, N. ${ }^{\circ} 12$, pp. 591-594, 2007

\title{
Fracaso renal agudo por microangiopatía trombótica asociado a enfermedad de Castleman
}

\author{
A. LÓPEZ MONTES, E. ANDRÉS MOMPEÁN, M. MARTÍNEZ VILLAESCUSA, \\ F. LLAMAS FUENTE, M. L. GARCÍA-MAURIÑO GUELBENZU ${ }^{1}$
}

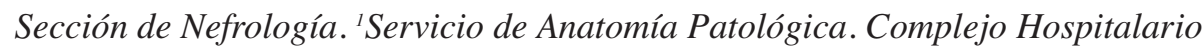

Universitario. Albacete

ACUTE RENAL FAILURE DUE TO THROMBOTIC MICROANGIOPATHY ASSOCIATED WITH CASTLEMAN'S DISEASE

\begin{abstract}
RESUMEN
Publicamos un caso de un paciente de 30 años que acude al servicio de Urgencias con dolor abdominal, fiebre, inestabilidad hemodinámica, hepatoesplenomegalia, fracaso renal agudo anúrico, linfadenopatías latero-cervicales, anemia y trombocitopenia. El paciente fue tratado con antibióticos empíricos, esteroides, gammaglobulinas, noradrenalina y hemodiálisis intermitente diaria con buena respuesta. La biopsia renal mostró datos de microangiopatía trombótica y tras la biopsia de una adenopatía se diagnosticó de enfermedad de Castleman.

La enfermedad de Castleman, también conocida como hiperplasia nodular linfática gigante o angiofolicular, es una entidad clínico-patológica de etiología desconocida. La afectación renal es heterogénea y frecuente.
\end{abstract}

PALABRAS CLAVE: Riñón. Enfermedad de Castleman. Microangiopatía trombótica.

\begin{abstract}
We report a case of a 30-year-old man presenting with abdominal pain, fever, homodynamic instability, hepatosplenomegaly, acute renal failure, cervical lymph nodes, anaemia and thrombocytopenia. The patient was treated with empiric antibiotics, high dose corticosteroids, gammaglobulins, noradrenalin and diary intermittent haemodialysis, with an excellent response. The renal biopsy showed a thrombotic microangiopathy, the lymph node biopsy showed a Castleman's disease.

Castleman's disease (also known as giant lymph node hyperplasia or angiofollicular lymph node hyperplasia) is a clinicopathological entity of unknown aetiology. A number of renal alterations have been described in association with the Castleman's disease.
\end{abstract}

KEY WORDS: Kidney. Castleman's disease. Thrombotic microangiopathy.

López Montes A, Andrés Mompeán E, Martínez Villaescusa M, Llamas Fuente F, García-Mauriño Guelbenzu ML. Fracaso renal agudo por microangiopatía trombótica asociado a enfermedad de Castleman. An Med Interna (Madrid) 2007; 24: 591-594.

\section{INTRODUCCIÓN}

La enfermedad de Castleman es una alteración linfoproliferativa infrecuente también conocida como hiperplasia nodular linfoide angiofolicular o hiperplasia nodular linfoide gigante (1), de etiología desconocida, que fue descrita por primera vez en 1956 por Castleman. Se trata de una rara alteración clasificada dentro de las enfermedades linfoproliferativas atípicas (1). Existen dos tipos de enfermedad de Castleman, el tipo hialino vascular $(90 \%)$ y el tipo de células plasmáticas (10\%) (2). La enfermedad de Castleman se considera resultado de una estimulación crónica antigénica debida a un estado inflamatorio o infeccioso desconocido (3) o como resultado de un defecto de la regulación del sistema inmune (4).

Presenta un curso muy heterogéneo, desde síntomas leves y persistentes en algunos casos durante más de 15 años (5) hasta casos con una progresión fatal. La afectación renal es muy heterogénea y relativamente frecuente, pudiendo afectar hasta el $54 \%$ de los casos (6), apareciendo proteinuria con o sin hematuria en el $80-90 \%$ de los casos de enfermedad de Castleman multicéntrica (4). Publicamos el caso de un paciente con fracaso renal agudo por microangiopatía trombótica, trombopenia e inestabilidad hemodinámica en el seno de una enfermedad de Castleman.

\section{CASO APORTADO}

Paciente varón de 30 años que ingresa en nuestra Unidad de Nefrología por fracaso renal anúrico acompañado de fiebre y trombocitopenia, con inestabilidad hemodinámica. Previamente asintomático y sin antecedentes de interés hasta el comienzo de un cuadro de epistaxis, diarrea, dolor abdominal y fiebre de 15 días de evolu-

Trabajo aceptado: 27 de julio de 2007 
ción. El examen físico mostraba datos de sobrecarga hídrica con edemas generalizados, hepatoesplenomegalia y linfadenopatías laterocervicales, supraclaviculares, inguinales y axilares, con un abdomen distendido. La presión arterial era 100/50 $\mathrm{mmHg}$, temperatura $38,5^{\circ}$ $\mathrm{C}$ y la frecuencia cardiaca de 85 latidos minutos/min. En los datos de laboratorio destacaba: Urea $173 \mathrm{mg} / \mathrm{dl}$, creatinina $4 \mathrm{mg} / \mathrm{dl}$, enzimas hepáticas normales, hemoglobina $10 \mathrm{~g} / \mathrm{dl}$, plaquetas $25.000 / \mathrm{mm}^{3}$, leucocitos $8.400 / \mathrm{mm}^{3}$, porcentaje de reticulocitos $3 \%$, velocidad de sedimentación globular $55 \mathrm{~mm} / \mathrm{h}$, proteína $C$ reactiva $325 \mathrm{mg} / \mathrm{L}$, tiempo de tromboplastina parcial activada $33 \mathrm{~s}$, actividad de protrombina $85 \%$, fibrinógeno $347 \mathrm{mg} / \mathrm{dl}$, productos de la degradación de la fibrina 40, lactato deshidrogenasa $325 \mathrm{UI} / \mathrm{L}$, bilirrubina 1,2 $\mathrm{mg} / \mathrm{ml}$, haptoglobina 225 , test de Coomb's directo e indirecto negativos. El frotis de sangre periférica mostraba la presencia escasa de algún hematíe fragmentado. La biopsia de médula ósea fue normal, con megacariocitos y el proteinograma mostró hiperglobulinemia policlonal alfa $1-\mathrm{G}$ y alfa $2-\mathrm{G}$. En orina: cilindros hialinos y granulosos, proteinuria $0,3 \mathrm{~g}$ /día. En la radiografía de tórax se apreciaba una cardiomegalia con derrame pleural bilateral y leve ensanchamiento mediastínico, el ecocardiograma mostró un derrame pericárdico moderado. La ecografía renal fue normal. La serología de virus de hepatitis B, C, VIH, Hantavirus, Brucella, Toxoplasma, Cytomegalovirus, virus herpes simplex, virus Epstein-Barr, Leptospira fue negativa. Los anticuerpos (Ac) antinucleares (ANA), Anti DNA, Ac antimembrana basal, crioglobulinas, Ac contra citoplasma de neutrófilos (ANCA), C3, C4, y la proteína Bences Jones en orina fueron normales o negativos. La biopsia renal mostró lesiones compatibles con microangiopatía trombótica con afectación exclusivamente glomerular, sin lesiones a nivel de vasos extraglomerulares y sin datos de necrosis tubular aguda. La biopsia de tejido linfoide mostró folículos linfoides abundantes en toda su sección con centros involucionados y con pequeños vasos capilares penetrando hacia su interior. Entre los folículos había abundantes capilares con endotelios prominentes así como una leve plasmocitosis policlonal, hiperplasia de células dendríticas y ausencia de células B intrafoliculares compatible con enfermedad de Castleman tipo hialino-vascular, con detección del virus del herpes 8 negativa. En el momento agudo el paciente requirió tratamiento con hemodiálisis diaria intermitente, precisando tratamiento con noradrenalina por inestabilidad hemodinámica. Se administró gammaglobulinas, así como esteroides intravenosos (125 mg metilprednisolona durante 5 días) y antibioterapia empírica, con resolución del cuadro renal y leve mejoría de la trombocitopenia.

Posteriormente y tras el diagnóstico de la enfermedad linfoproliferativa el paciente fue tratado con 6 ciclos de CHOP (ciclofosfamida, doxorubicina, vincristina y prednisolona) y Rituximab con normalización de las alteraciones analíticas y remisión completa de la enfermedad que se ha mantenido a lo largo de los últimos 3 años.

\section{DISCUSIÓN}

Describimos un caso de enfermedad de Castleman tipo hialino-vascular multicéntrica asociado a múltiples manifestaciones sistémicas, incluida la afectación renal severa, en forma de microangiopatía trombótica, la cual es una complicación extremadamente rara en este cuadro que evidencia una alteración en la regulación inmunológica de estos pacientes. Previamente se han descrito casos de microangiopatía trombótica asociado a enfermedades neoplásicas, la mayoría de las veces asociado a cuadros metastáticos de adenocarcinomas (6), con un pronóstico fatal. La enfermedad puede ser localizada (unicéntrica), apareciendo con más frecuencia en adultos jóvenes con localización preferentemente mediastínica (60$75 \%$ ), cuello (20\%) o intra-abdominal (10\%), o multicéntrica, mostrando linfadenopatías generalizadas y afectación sistémi- ca, siendo menos frecuente, más agresiva y afectando a pacientes en la sexta década de la vida (7), correspondiendo en la mayoría de las ocasiones a la variante de células plasmáticas. El tipo hialino-vascular constituye el 80-90\% de los casos y la variante de células plasmáticas el 10\%, encontrándose en ocasiones datos histológicos de las dos variantes en un mismo nódulo linfático lo que se conoce cómo variante mixta de la enfermedad de Castleman. La enfermedad de Castleman se ha asociado a pacientes VIH con sarcoma de Kaposi infectados por el virus herpes 8 , detectándose el virus del herpes 8 en el $40 \%$ de los pacientes con la forma multicéntrica (8). También se ha descrito un aumento en la incidencia del linfoma de Hodgkin en pacientes con enfermedad de Castleman (9).

El mecanismo de producción de la enfermedad de Castleman no está claro. Existe evidencia clínica y experimental que concede a la interleuquina 6 (IL-6) producida por las células B del centro germinal de los nódulos linfáticos hiperplásicos un papel primordial en la patogénesis de la enfermedad (10). IL6 transgénica introducida en ratones de experimentación provoca anormalidades como hipergammaglobulinemia, trombocitosis, infiltración inflamatoria en los tejidos, proliferación de células mesangiales en el riñón, esplenomegalia y linfadenopatías, además de una plasmocitosis semejante a la encontrada en la enfermedad de Castleman $(11,12)$. La IL-6 causa proliferación y diferenciación de la células B en células productoras de anticuerpos, además aumenta la secreción del factor de crecimiento derivado del endotelio vascular (VEGF), factor implicado también en la patogénesis de la enfermedad de Castleman $(13,14)$. Las plaquetas son trasportadoras de dicho factor, la liberación de VEGF aumenta la permeabilidad vascular y la actividad coagulante plasmática en vivo. En un caso descrito por Seida et al (15) se detectó una importante elevación de VEGF y el paciente, al igual que el nuestro, mostró trombocitopenia y coagulación intravascular diseminada. Mediante inmuno-marcaje de VEFG, demostraron que los nódulos linfáticos con proliferación de células plasmáticas eran uno de los sitios de mayor producción de VEGF, explicando la aparición de derrame pleural, la ascitis, el edema generalizado y la aparición de proteinuria por el aumento de la permeabilidad vascular. Por otra parte también se le ha implicado en un cierto papel protector de la microangiopatía trombótica. Recientemente, VEGF ha sido implicado en la protección e incluso en la aceleración de la recuperación del daño renal de la microangiopatía trombótica inducida por anticuerpos contra el endotelio en modelos experimentales en ratas $(16,17)$. Así, el daño endotelial y la microangiopatía trombótica estarían inducidas por la enfermedad de Castleman y posteriormente la producción local de VEGF podría proteger y acelerar la recuperación del daño glomerular (15).

En general los pacientes con enfermedad de Castleman presentan a menudo malestar general, fiebre, anorexia, sudoración nocturna y perdida de peso. A la exploración suelen presentar linfadenopatías múltiples, hepatoesplenomegalia, ascitis, edemas, derrame pleural y pericárdico. En los datos de laboratorio suele destacar trombopenia, anemia, hipoalbuminemia e hipergammaglobulinemia. En ocasiones llegan a presentar, como en nuestro paciente, datos de shock que requiere su ingreso en unidades de cuidados intensivos. Muchos de estos síntomas se atribuyen al aumento de IL-6.

La afectación renal no es infrecuente, habiéndose descrito casos de glomerulonefritis membranosa (18), amiloido- 
sis $(19,20)$, nefritis intersticial (21) glomerulonefritis proliferativa mesangial (22), glomerulonefritis membrano-proliferativa (23-25), glomerulonefritis rápidamente progresiva (26), enfermedad por cambios mínimos (27), glomerulonefritis fibrilar (27), o fallo renal agudo secundario a microangiopatía trombótica $(19,29,30)$.

La regresión de los síntomas asociados y de la alteración renal ocurre tras la desaparición del las masas linfoides (6).

El tratamiento de la enfermedad de Castleman multicéntrica es controvertido. La resección parcial de la masa tumoral puede mejorar la sintomatología en algunos casos, pero no aporta beneficio a largo plazo (6) excepto en la variedad hialino-vascular localizada con raras recurrencias tras la cirugía $(31,32)$. Dosis bajas de radioterapia puede aportar beneficio en casos limitados $(32,33)$. El uso de esteroides solos o junto con agentes quimioterápicos presenta resultados conflictivos (33). En pacientes inmunocompetentes se han utilizado regimenes quimioterápicos basados en la experiencia en linfomas con CHOP. En pacientes VIH el tratamiento se basa principalmente en el uso de agentes antirretrovirales. El Interferón alfa ha sido administrado con o sin antirretrovirales o agentes quimioterápicos para inducir remisión o como tratamiento de mantenimiento $(34,35)$. Así como anticuerpos anti-IL-6 monoclonal (36) y más recientemente el anti CD-20 monoclonal Rituximab asociado a quimioterapia (37).

Nuestro paciente presentó un cuadro de inestabilidad hemodinámica con datos de aumento de permeabilidad vascular como la ascitis y el derrame pericárdico, pero sin claros datos de anemia hemolítica que hiciera sospechar un cuadro de síndrome hemolítico urémico, por lo que no se planteó el uso de plasmaféresis. Probablemente la inestabilidad hemodinámica contribuyera a la aparición del daño renal aunque no se encontraron hallazgos histológicos de una necrosis tubular aguda. El tratamiento empírico con esteroides pudo contribuir a la resolución del cuadro renal.

\section{Bibliografía}

1. Castleman B, Iverson K, Menéndez VP. Localized mediastinal lymphnode hyperplasia resembling thymoma. Cancer 1956; 9: 822-830.

2. Keller AR, Hochholzer L, Castleman B. Hyaline-vascular and plasmacell types of giant lymph node hyperplasia of the mediastinum and other locations. Cancer 1972; 29: 670-683.

3. Said R, Tarawneh M. Membranoproliferative glomerulonephritis associated with multicentric angiofollicular lymph node hyperplasia. Case report and review of the literature. Am J Nephrol 1992; 12: 466-470.

4. Peterson BA, Frizzera G. Multicentric Castleman's disease. Semin Oncol 1993; 20: 636-647.

5. Moir DH, Choy T, Dalton WR. Giant lymph node hyperplasia: persistence of symptoms for 15 years. Cancer 1982; 49: 748-750.

6. Frizzera G, Peterson BA, Bayrd ED, Goldman A. A systemic lymphoproliferative disorder with morphologic features of Castleman's disease: clinical findings and clinicopathologic correlations in 15 patients. J Clin Oncol 1985; 3: 1202-1216.

7. Waterston A, Bower M. Fifty years of multicentric Castleman's disease. Acta Oncol 2004; 43: 698-704.

8. Lachant NA, Sun NC, Leong LA, Oseas RS, Prince HE. Multicentric angiofollicular lymph node hyperplasia (Castleman's disease) followed by Kaposi's sarcoma in two homosexual males with the acquired immunodeficiency syndrome (AIDS) Am J Clin Pathol 1985; 83: 27 33.

9. Oksenhendler E, Boulanger E, Galiecer L. High incidence of Kaposi sarcoma-associated herpesvirus-related non-Hodgkin lymphoma in patients with HIV infection and multicentric Castleman disease. Blood 2002; 99: 2331-2336.

10. Beck JT, Hsu SM, Wijdenes J, Bataille R, Klein B et al. Brief report: alleviation of systemic manifestations of Castleman's disease by monoclonal anti-interleukin-6 antibody. N Engl J Med 1994; 330: 602-605.

11. Nishimoto N, Kishimoto T, Yoshizaki K. Anti-interleukin 6 receptor antibody treatment in rheumatic disease. Ann Rheum Dis 2000; 59 (Supl. 1): i21-27.

12. Aoki Y, Jaffe ES, Chang Y. Angiogenesis and hematopoiesis induced by Kaposi's sarcoma-associated herpesvirus-encoded interleukin-6 Blood 1999; 93: 4034-4043

13. Nishi J, Maruyama I. Increased expression of vascular endothelial growth factor (VEGF) in Castleman's disease: proposed pathomechanism of vascular proliferation in the affected lymph node. Leuk Lymphoma 2000; 38: 387-94.

14. Foss HD, Araujo I, Demel G, Klotzbach H, Hummel M, Stein H. Expression of vascular endothelial growth factor in lymphomas and Castleman's disease. J Pathol 1997; 183: 44-50.

15. Seida A, Wada J, Morita Y, Baba M, Eguchi J, Nishimoto N et al. Multicentric Castleman's disease associated with glomerular microangio- pathy and MPGN-like lesion: does vascular endothelial cell-derived growth factor play causative or protective roles in renal injury? Am J Kidney Dis 2004; 43: E3-9.

16. Kim YG, Suga SI, Kang DH, Jefferson JA, Mazzali M, Gordon KL et al. Vascular endothelial growth factor accelerates renal recovery in experimental thrombotic microangiopathy. Kidney Int 2000; 58: 23902399.

17. Suga S, Kim YG, Joly A, Puchacz E, Kang DH et al. Vascular endothelial growth factor (VEGF121) protects rats from renal infarction in thrombotic microangiopathy. Kidney Int 2001; 60: 1297-1308.

18. Ruggieri G, Barsotti P, Coppola G, Spinelli C, Balducci A et al. Membranous nephropathy associated with giant lymph node hyperplasia. A case report with histological and ultrastructural studies. Am J Nephrol 1990; 10: 323-328.

19. Altiparmak MR, Pamuk GE, Pamuk ON, Dogusoy G. Secondary amyloidosis in Castleman's disease: review of the literature and report of a case. Ann Hematol 2002; 81: 336-339.

20. Curioni S, D'Amico M, Quartagno R, Martino S, Dell'Antonio G, Cusi D. Castleman's disease with nephrotic syndrome, amyloidosis and autoimmune manifestations. Nephrol Dial Transplant 2001; 16: 14751478.

21. Tsukamoto Y, Hanada N, Nomura Y, Hiki Y, Kasai K, Shigematsu H, et al. Rapidly progressive renal failure associated with angiofollicular lymph node hyperplasia. Am J Nephrol 1991; 11: 430-436.

22. Combe C, Bouchet JL, Potaux L, Morel D, Deminiere C, et al. Castleman's disease and glomerular nephropathy (apropos of 1 case)Nephrologie 1989; 10: 163-166.

23. Said R, Tarawneh M. Membranoproliferative glomerulonephritis associated with multicentric angiofollicular lymph node hyperplasia. Case report and review of the literature. Am J Nephrol 1992; 12: 466-470.

24. Akimoto H, Shirai M, Usutani S, Masaoka H, Hidaka S, Kakuta S, et al. Membranoproliferative glomerulonephritis-like lesion with fibrillary deposition associated with multicentric Castleman's disease. Nippon Jinzo Gakkai Shi 1998; 40: 301-308.

25. Uthup S, Balachandran K, Anmal VA, Salam RA, George J, Nair GM, et al. Renal involvement in Multicentric Castleman Disease with glomeruloid hemangioma of skin and plasmocytoma. Am J Kid Dis 2006; 48: E17-E24.

26. Furuichi K, Wada T, Shimizu M, Segawa C, Ohta S et al. Antimyeloperoxidase-antibody-positive rapidly progressive glomerulonephritis associated with Castleman's disease. Nephrol Dial Transplant 1998; 13: 1556-1558.

27. Frizzera G, Banks PM, Massarelli G, Rosai J. A systemic lymphoproliferative disorder with morphologic features of Castleman's disease. Pathological findings in 15 patients. Am J Surg Pathol 1983; 7: 211-231. 
28. Miadonna A, Salmaso C, Palazzi P, Elli A, Braidotti P, Lambertenghi Deliliers G. Fibrillary glomerulonephritis in Castleman's disease. Leuk Lymphoma 1998; 28: 429-435.

29. Lajoie G, Kumar S, Min KW, Silva FG. Renal thrombotic microangiopathy associated with multicentric Castleman's disease. Report of two cases. Am J Surg Pathol 1995; 19: 1021-1028.

30. Jones CH, Will EJ. Angiofollicular lymph node hyperplasia, accelerated hypertension, and acute renal failure: a case report. Nephrol Dial Transplant 1996; 11: 352-354.

31. Moore PS, Chang Y. Detection of herpesvirus-like DNA sequences in Kaposi's sarcoma lesions from persons with and without HIV infection. New Engl J Med 1995; 332: 1181-1185.

32. Dupin N, Fisher C, Kellam P. Distribution of humana herpesvirus 8 latently infected cells in Kaposi's sarcoma, multicentric Castleman's disease and primary effusion lymphoma. Proc Natl Acad Sci USA 1999; 96: 4546-4551.
33. Borne WB, Lewis JJ, Filippa DA, Niesvizky R et al. The management of unicentric and multicentric Castleman's disease: a report of 16 cases and a review of the literature. Cancer 1999; 85: 706-717.

34. Strohal R, Tschachler E, Breyer S. Reactivation of Behcet's disease in the course of multicentric HHV8-positive Castleman's disease: long-term complete remission by a combined chemo/radiation and interferon-alpha therapy regimen. Br J Haematol 1998; 103: 788790 .

35. Nord JA, Karter D. Low dose interferon-alpha therapy for HIV-associated multicentric Castleman's disease. Int J STD AIDS 2003; 14:6162 .

36. Katsume A, Saito H, Yamada Y. Anti-interleukin 6 (iL-6) receptor antibody suppresses Castleman's disease like symptoms emerged in iL6 transgenic mice. Cytokine 2002; 20: 304-311.

37. Avivi I, Robinson S, Goldstone A. Clinical use of rituximab in haematological malignancies. Br J Cancer 2003; 89: 1389-1394. 